\title{
Investigation on the COVID-19 Outbreak in India: Lockdown Impact and Vulnerability Analysis
}

\author{
Nilay Nishant", Avinash Chouhan, Dibyajyoti Chutia, Puyam S. Singh, Sanjiv Ojah, Sanjiban Roy, \\ P. L. N. Raju
}

North Eastern Space Applications Centre, Department of Space, Umiam, Meghalaya, India

Email: *nilay.nishant@nesac.gov.in

How to cite this paper: Nishant, N., Chouhan, A., Chutia, D., Singh, P.S., Ojah, S., Roy, S. and Raju, P.L.N. (2020) Investigation on the COVID-19 Outbreak in India: Lockdown Impact and Vulnerability Analysis. Journal of Geographic Information System, 12, 334-347.

https://doi.org/10.4236/jgis.2020.124021

Received: July 8, 2020

Accepted: August 9, 2020

Published: August 12, 2020

Copyright ( 2020 by author(s) and Scientific Research Publishing Inc. This work is licensed under the Creative Commons Attribution International License (CC BY 4.0).

http://creativecommons.org/licenses/by/4.0/

\begin{abstract}
Coronavirus (COVID-19) disease is a major pandemic which has taken the world by storm. More than 524,000 citizens of the globe have succumbed to the disease as on $3^{\text {rd }}$ July, 2020. Accurate modeling of the dynamics of the disease spread is required to curb the virus. With the availability of large amount of data made available publicly by the Government agency as well as the other live crowdsourcing media, it is possible to develop an accurate local prediction tool. In this study, we analyze the dynamics of local outbreaks of COVID-19 for few states of India with major outbreaks and for India as a whole. The large amount of data available from the COVID-19 Tracker India platform was utilized to estimate the impact of lockdown in the country and the states with major outbreaks. The effectiveness of the lockdown implementation by the respective states is studied for the analysis. The lockdown was categorized into strict, moderate, and lenient. The model is deployed on a web based platform to disseminate the alert to the public. We further extend the ordinary differential Equation (ODE) based model to generate district level vulnerability index for the whole country based on the rate of change of infected people, breach in social distancing, and population. Around $47 \%$ of districts of the country were not found vulnerable; however, $13 \%$ of the districts were identified as high risk for the disease outbreak.
\end{abstract}

\section{Keywords}

COVID-19, Corona India, Time Series Analysis, SIR Model, Vulnerability Analysis 


\section{Introduction}

The Novel Coronavirus was first reported in India in January 2020. Since then, it has engulfed more than 628,000 people in the country, and more than 10,993,124 globally out of which over 52.400 citizens have succumbed to the pandemic disease as on July 3, 2020 [1]. After first emerging in late January, the number of infected people in India remained constant until the beginning of March, post which, an exponential growth was observed [2]. Since the COVID-19 disease does not have medicinal cure as of yet [3] [4], one of the major approaches followed by the countries to break the chain of transmission around the world remains to be "social distancing" and imposing an area-wise, state-wise or nationwide lockdown. Since its outbreak in Wuhan, China, nearly all the countries which have been affected by this pandemic are imposing lockdown to control this vicious disease. As on 24th March 2020, Government has issued a nationwide lockdown to contain the pandemic. During the lockdown period citizens are required to maintain social distancing and travelling is banned. The lockdown was imposed in four phases: Phase-1: 25 March 2020-14 April 2020 (21 days); Phase-2:15 April 2020-3 May 2020 (19 days); Phase-3: 4 May 2020-17 May 2020 (14 days); Phase-4: 18 May 2020-31 May 2020 (14 days). The total number of cases had reached 190,648 with 5405 deaths of the widely spreading deadly disease by the end of lockdown i.e. $31^{\text {st }}$ May 2020. The Government has started the procedure of lifting off the lockdown as an initiative to bring normalcy across the country since June 1, 2020. In order to efficiently implement the lockdown, it is imperative for the Government to have accurate forecasts as well as the projections of the disease spread including identification of the hotspots and vulnerable areas.

At the initial stages of the outbreak, most of the pandemics follow an exponential curve that eventually flatten out over the period of time. With the change in the growth rate, the exponential curve eventually turns into a logistic curve. Extrapolating the trends based on current growth rates will lead to inaccurate results [6]. Susceptible-infectious-recovered (SIR) model [7] is used to include considerations for susceptibility, infectious, and recovered or deceased individuals. These models have shown a significant predictive ability for the growth of COVID-19 [6]. Social distancing plays an important role in containing outbreak of COVID-19 cases. The impact of lockdown can be visualized by plotting the $\log$ (new daily reported cases) against log (Cumulative confirmed cases) as in Figure 1 and can be clearly observed evidently for countries like South Korea, China, and Australia. Countries like Germany, Italy and Spain have shown early signs of flattening the curve.

WebGIS is an effective approach to provide geographic information to a wide range of users. It has modified the traditional way of using GIS as a database-mapping and spatial analytical tool. In addition, WebGIS promotes the sharing and synthesis of multisource data and enables widespread sharing of spatial data and geosciences models. Web based analysis for spatio temporal 


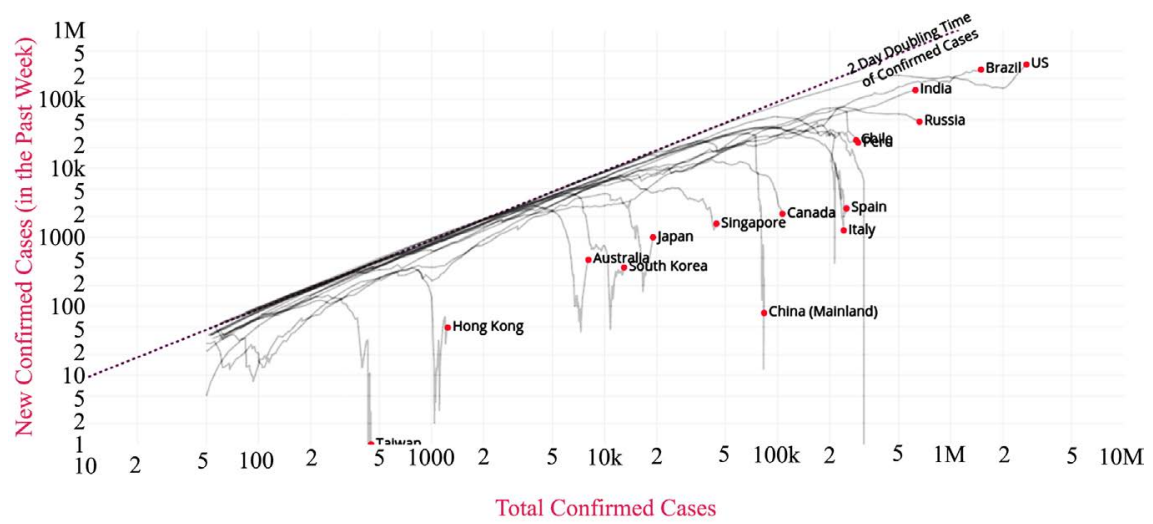

Figure 1. Impact of Lockdown on COVID-19 spread for different countries [5].

analysis has been around for more than a decade. The basic architecture of a web-based GIS application usually includes a web server in server-side that can handle incoming requests and generate corresponding responses.

Since the advent of COVID-19 many studies have been reported addressing the forecasting of the disease, however very few studies have addressed the impact of lockdown, by quantifying the social distancing parameters using the crowdsourced information. Considering the impact of dynamics of the virus on the country, following objectives are addressed in this study.

- Impact of lockdown on the outbreak of COVID-19 in India and forecasting the COVID-19 scenario using SIR model for the whole country.

- Computing District-wise vulnerability analysis based on crowd-sourced information and modified SEIR model.

- Disseminated of information using a WebGIS framework which is developed using open-source tools and technologies.

\section{Methods}

\subsection{COVID-19 Outbreak for Multiple Lockdown Scenarios}

SIR model is an epidemiological model that accounts for a number of susceptible $(S)$, number of infectious $(I)$ and the number of recovered or deceased (or immune) individuals $(R)$. Countries which are already in phase-II and phase-III of the pandemic are using the same model to estimate the outbreak [8]-[14]. Initially, the growth is exponential for countries where the outbreak is in initial stages which are followed by a logistic curve for countries where the growth rate has stabilized (such as China and South Korea). SIR model was used to model the spread of disease and make forecasts.

We further implement a modified susceptible-exposed-infected-recovered (SEIR) model which includes the parameters of social distancing to derive the vulnerable areas. This information is vital as breach in social distancing plays an important role in the outbreak of the disease. It is therefore necessary to estimate not only the total number of infections but also the pattern of distribution across 
the districts based on the population and rate of change in the number of infected people. The study presents a fully data dependent model to estimate the effectiveness of the lockdown in the states of India with major outbreaks during the lockdown period. Projections are made for three lockdown scenarios, i.e. lenient, moderate and strict lockdown. The model also does not take into account other factors such as immunity of population to the disease [9], impact of weather change on outbreak of disease.

$$
\begin{gathered}
S^{\prime}=-\beta S I \\
I^{\prime}=\beta S I-\gamma I \\
R^{\prime}=\gamma I \\
N=S+I+R
\end{gathered}
$$

where $\beta$ is the transmission rate, and $\gamma$ is the average recovery rate. It is further observed from Equation (2) that the change in the infected people is dependent on $\beta$ and $\gamma$. The number of recovered or deceased (or immune) individuals is estimated based on Equation (3) and is dependent on the average recovery rate. The model assumes that during the duration of simulation, the population remains constant.

In this part, we fit the SIR model with available COVID-19 data from India for the states with major COVID-19 outbreaks. We estimate $\beta$ and $\gamma$, which are significant parameters in governing the dynamics of outbreak. $\beta$ and $\gamma$ are estimated by minimizing the RMSE between the predicted values and the actual values, but also are specific to each country.

Another essential factor for computing is So, i.e. Initial population, which is susceptible to the infection. The current study, we consider three scenarios, i.e. lenient lockdown, strict lockdown and Moderate Lockdown. S0 is computed for the three scenarios separately. It is estimated based on the following assumptions. Since Europe is currently recovering as cases have started to decline, similarly in the Asian countries, based on the peak active cases and the total population of European countries, we consider $0.5 \%$ of the total population as the upper cap of the population being affected by COVID-19. Considering the factors such as early lockdown and mean age of the total population is less than that of European countries, initially exposed population is from Table 1 . S0 for moderate lockdown is assumed as 50\% of initially exposed population whereas $\mathrm{S} 0$ for the strict lockdown scenario is considered as $25 \%$ of S0. This information was calculated individually for each state with major outbreaks and ingested into SIR model.

Finally, the projections are made based on the model and root mean square error (RMSE) is computed for all the three scenarios of lockdown. To further assess the severity of the COVID-19 outbreak within states, state-wise information was plotted for all the states with major outbreak of COVID-19 cases. Scenario with the minimum RMSE is considered as the representative lockdown scenario of the state. The assessment of the change in the lockdown scenario is 
Table 1. Initially exposed population for states with major outbreak\& RMSE for different lockdown scenarios.

\begin{tabular}{ccccc}
\hline \multirow{2}{*}{ State } & Population & \multicolumn{3}{c}{ RMSE } \\
\cline { 3 - 5 } & Exposed (S0) & Lenient & Moderate & Strict \\
\hline Maharashtra (MH) & 57,100 & 177.83 & 122.40 & 137.91 \\
Gujarat (GJ) & 31,350 & 82.66 & 81.44 & 88.32 \\
Rajasthan (RJ) & 34,450 & 82.00 & 77.28 & 71.92 \\
Delhi (DL) & 9437 & 173.17 & 121.10 & 391.03 \\
Madhya Pradesh (MP) & 36,650 & 99.18 & 89.36 & 80.50 \\
Uttar Pradesh (UP) & 55,661 & 49.80 & 56.39 & 54.19 \\
Tamil Nadu (TN) & 33,950 & 179.27 & 178.65 & 166.31 \\
Andhra Pradesh (AP) & 24,850 & 35.54 & 38.69 & 33.77 \\
West Bengal (WB) & 45,145 & 23.45 & 22.89 & 22.65 \\
Punjab (PB) & 14,000 & 39.71 & 27.75 & 28.01 \\
\hline
\end{tabular}

estimated by taking the difference between the observed value and the scenario projection. The scenario with the minimum difference value at a particular date is considered as the representative scenario for the respective state.

\subsection{Maintaining the Integrity of the Specifications}

SEIR model is an extension of SIR model which is a widely used epidemiological model for modeling disease spread through the population. It is the acronym for Susceptible, Exposed, Infected and Recovered. The model simulates the distribution of population in the four categories as mentioned above. In addition to the three parameters implemented i.e. $S, I$ and $R$, we have an additional parameter called $E$ which represents total exposed population. $E$ can be calculated from the Equation (6). Model can be depicted from the Equation below.

$$
\begin{gathered}
S^{\prime}=-\beta S I \\
E^{\prime}=\beta S I-\alpha E \\
I^{\prime}=\alpha E-\gamma I \\
R^{\prime}=\gamma I \\
N=S+E+I+R
\end{gathered}
$$

There is no change in estimating the Susceptible and the recovered population. It can be observed from Equation (6) that the estimation of exposed population is dependent on the contact rate and decreases based on the incubation period. Equation (7) gives us the change in infected people, it is dependent on the exposed population, incubation period and transmission rate. It decreases based on the infectious period. In addition to the parameters $\beta$ and $\gamma$, SEIR model has one additional parameter, $\alpha$ which represents inverse of the incubation period. 
In order to study the impact of lockdown on the spread of disease, social distancing scaling parameter $(\rho)$ was included into the model. The value of $\rho$ ranges from $0-1$ and is computed from number of times, breach in social distancing during lockdown as reported by crowd sourced platform such as Map My India's Covid-19 portal, Equations (5) and (6) can be modified into Equations (10) and (11) .

$$
\begin{gathered}
S^{\prime}=-\rho \beta S I \\
E^{\prime}=\rho \beta S I-\alpha E \\
E=\frac{\gamma I-I^{\prime}}{\alpha} \\
S=N-I-R-\frac{\gamma I-I^{\prime}}{\alpha} \\
V . I=\rho \beta\left(N-I-R-\frac{\gamma I-I^{\prime}}{\alpha}\right) * I-\alpha\left(\frac{\gamma I-I^{\prime}}{\alpha}\right)
\end{gathered}
$$

Considering the number of exposed people as a proxy for vulnerability, we can estimate it using the Equation (14) which is derived from SEIR model. The number of exposed people depends on population, change in number of infected people and the social distancing parameter (). The social distancing parameter is determined by normalizing the lockdown issues reported from various crowdsourced platforms. District level vulnerability is estimated by weighted distribution of exposed population of the state across the districts.

\subsection{System Architecture}

We have followed service oriented architecture for the development of WebGIS dashboard. It is a three tier i.e. Client, server and database (Figure 2). The landing page with semi-automated live data streaming features 1) to date and daily update on confirmed, active, recovered and death cases, 2) states showcasing hotspots, non-hotspots and non-infected districts, 3) vulnerability index map showing districts with very low, low, medium, high and very high susceptibility towards COVID-19, 4) Daily statistical charts on samples tested with age and gender-wise infection rate, 5) link to Assam, Meghalaya and Tripura dashboard (this can be customized by each state in India) 6) Predicted COVID-19cases based on SIR model. Currently, three web applications have been specifically designed and integrated on the top of the main COVID-19 dashboard to support

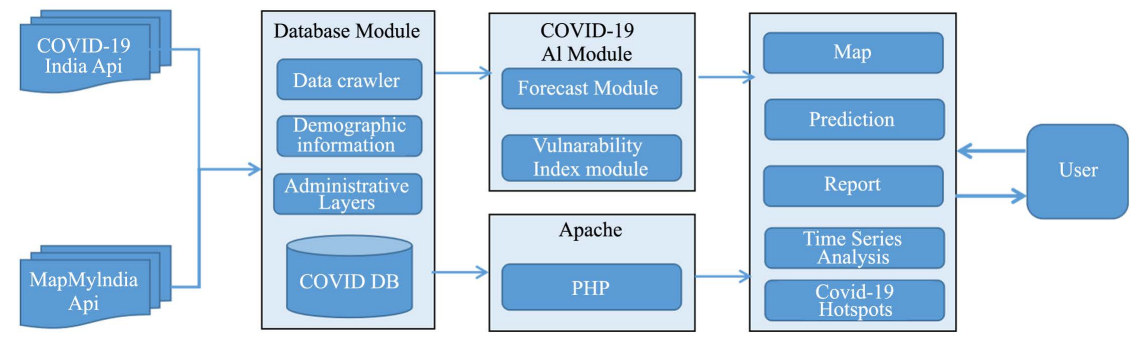

Figure 2. Architecture of COVID-19 decision support system. 
decision making activity using geospatial tools and services. It is equipped with state specific user applications with specific functionality such as 1) visualize facilities for possible expansion of quarantine centers along with details attributes, 2) address multi dimensional queries on the attributes of facilities, 3) visualize statistics based on custom polygon on the fly, 4) tack quarantine patients in real-time in a web based platform, 5) identify hotspots based on the number of patients and population distribution, 6) map quarantine patients at village level.

\section{Results and Discussions}

\subsection{COVID-19 Outbreaks during Lockdown}

The states with the major outbreaks were filtered out and SIR model projections were plotted on those states by minimizing the RMSE the COVID-19 outbreak. The projection details of the respective states can be observed from Table 1, states/union territories such as Gujarat, Delhi and Maharashtra are depicting major outbreak in the country. The projection details of the respective states can be observed from Table 1, states/union territories such as Gujarat, Delhi and Maharashtra are depicting major outbreak in the country. The inference can be cross validated from Figure 3; where the top 10 states with most number of the confirmed cases as on $31^{\text {st }}$ May, 2020 have been plotted. From Figure 3 it can be inferred that the curve for states like Maharashtra, Delhi and Gujarat seem to be rising exponentially. In contrast, Punjab on the other hand saw an unexpected spike in the number of patients due to the arrival of its citizens from Nanded, Maharashtra during the last week of April.

SIR model based projections for the whole country was carried out for three scenarios of lockdown (strict, moderate, and lenient). The results can be observed in Figure 4 and Figure 5. Based on the three scenarios, India will reach a peak in the number of cases by the May, 2020 with the total number of active cases being reported in India just under 70,000 active cases if strict lockdown was implemented across the country, in an event moderate lockdown was implemented the peak would be 100,000 and Lenient lockdown the peak will be

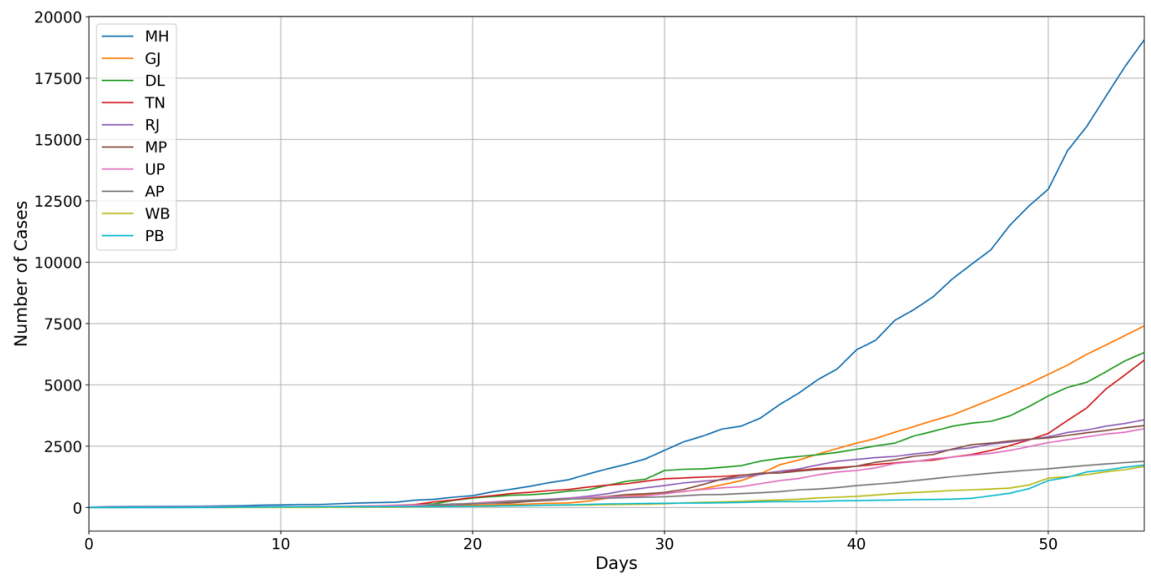

Figure 3. Number of confirmed cases for states with major outbreaks until 7-05-2020. 


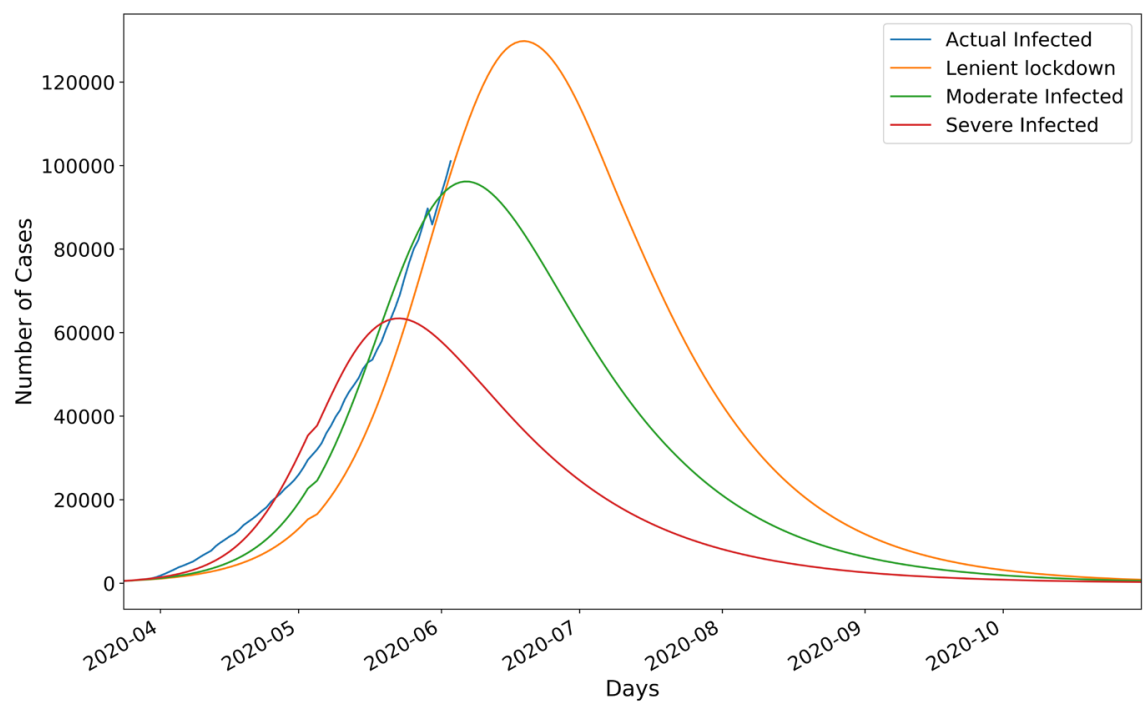

Figure 4. SIR model based projection of COVID-19 for India for three lockdown scenarios as on May 2020.

200,000. It is further observed that based on the current scenario, by the end of all the phases of lockdown, the country was following lenient lockdown as a whole. The peak in the case of moderate and lenient lockdown will be in the month of June. The projections were also extended to understand the gravity of the disease outbreak in the states of the country with major outbreak. The model is implemented for understanding the projections under the lockdown scenario i.e. until 31 May 2020. Post, implementation of unlocks i.e. from 1 June 2020, the dynamic parameters of SIR models have increased and it was possible to quantify the breach in the lockdown and movement of the passengers.

The results indicated in Table 1 convey that states such as Rajasthan, Tamil Nadu, Andhra Pradesh and West Bengal are following strict lockdown scenario whereas Delhi, Maharashtra and Gujarat are following moderate lockdown scenario, these are also the states with the most number of COVID-19 cases in the country as on 06 May 2020. Uttar Pradesh on the other hand might show a close correlation with lenient lockdown scenario, however on closer inspection it is observed that the difference between observed value and projected value for the three lockdown scenarios is minimum and thus also the shows that the observed value is very much under the projected value for strict lockdown scenario, hence Uttar Pradesh may be considered the following the path of strict lockdown.

Further, diving deep into the transition between the lockdown scenarios depicts that even though states such as Delhi, Punjab and Madhya Pradesh might be represented by strict or moderate lockdown scenarios, these states have transitioned into lenient lockdown scenario since the first week of May, 2020. The difference curve of Delhi also depicts that, the state has deviated from the strict lockdown scenario by second week of April, 2020; comparatively population density of Delhi is much higher than the other states. Maharashtra has also depicted the deviation for strict lockdown since last week of April, 2020. On the 

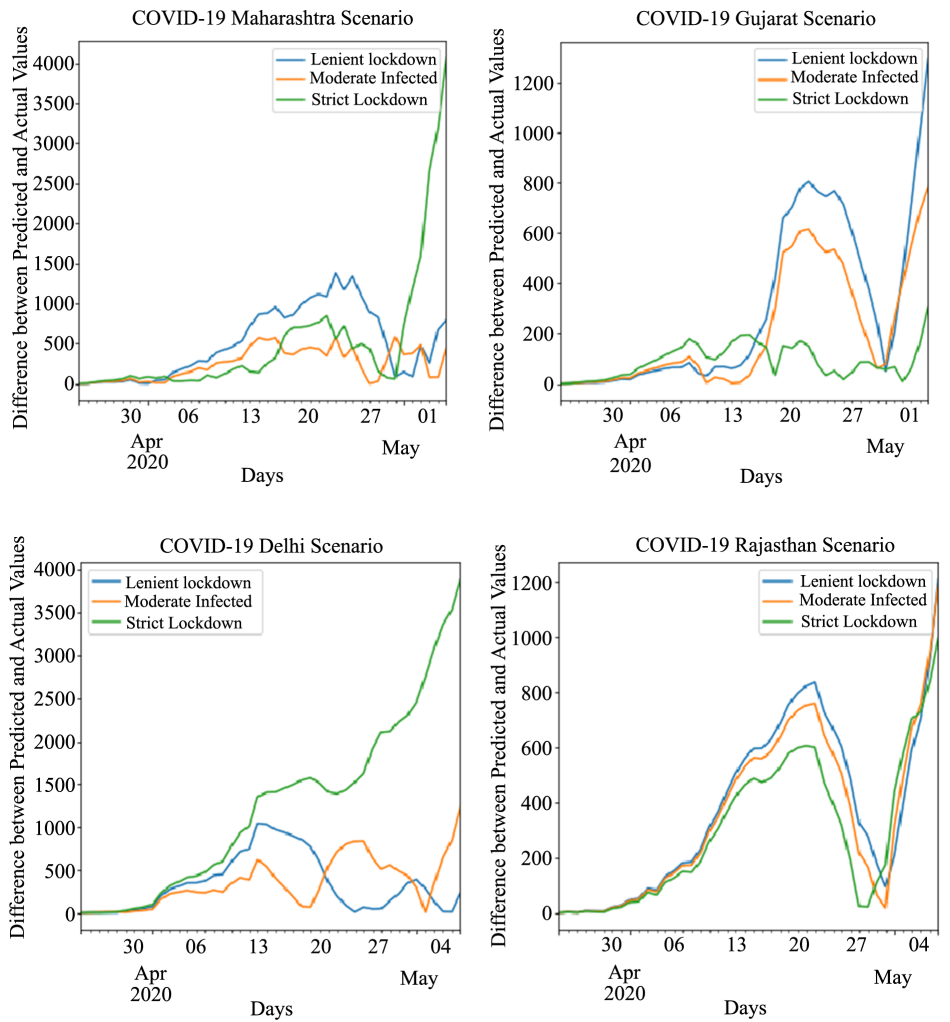

(a)
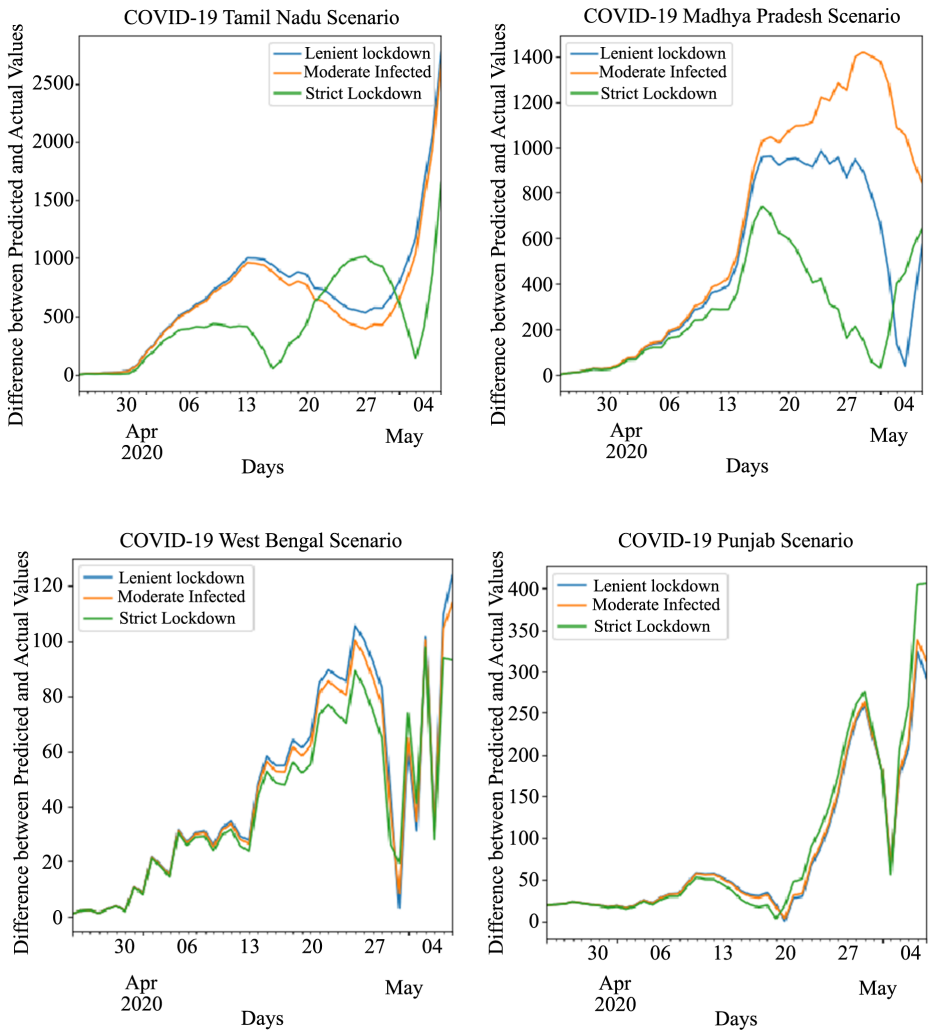

(b)

Figure 5. (a) Temporal change between lockdown scenarios with major outbreaks; (b) Temporal change between lockdown scenarios with major outbreaks, May 2020. 
other hand, the transition for all the other states depicts the close correlation between all the three scenarios to the observed values, these projections will get diverged in the month of May, 2020 as represented in Figure 4 \& Figure 5. Tamil Nadu is one of the states which had flattened the curve and the number of active cases had started to drop, however during the last week of April, 2020 the state was hit by a second wave of COVID-19, this can be observed in Figure 6. The deviation of the state from the strict lockdown during last week of April, 2020 is the representative of the second wave. The projections depicted in the three scenarios are based on the current situation of infected population and state of lockdown across the country, however if the scenario changes, the projections are subjected to change.

\subsection{Vulnerability Index}

District-wise vulnerability index is generated based on Equation (14). The vulnerability index is mainly dependent on the 3 factors. i.e. rate of change of infected people, social distancing and the population of the district. Figure 6 given below depicts the vulnerability index generated based on the confirmed data until 7th May, 2020. The breach in social distancing is quantified by estimating the total number of lockdown issues reported in each state via crowdsourcing. It can be observed from Figure 6 that majority of districts in Maharashtra, Rajasthan as well as Gujarat depicts high vulnerability. This can be seen as the also where districts with high count of infected people have reported higher numbers of breach in social distancing.

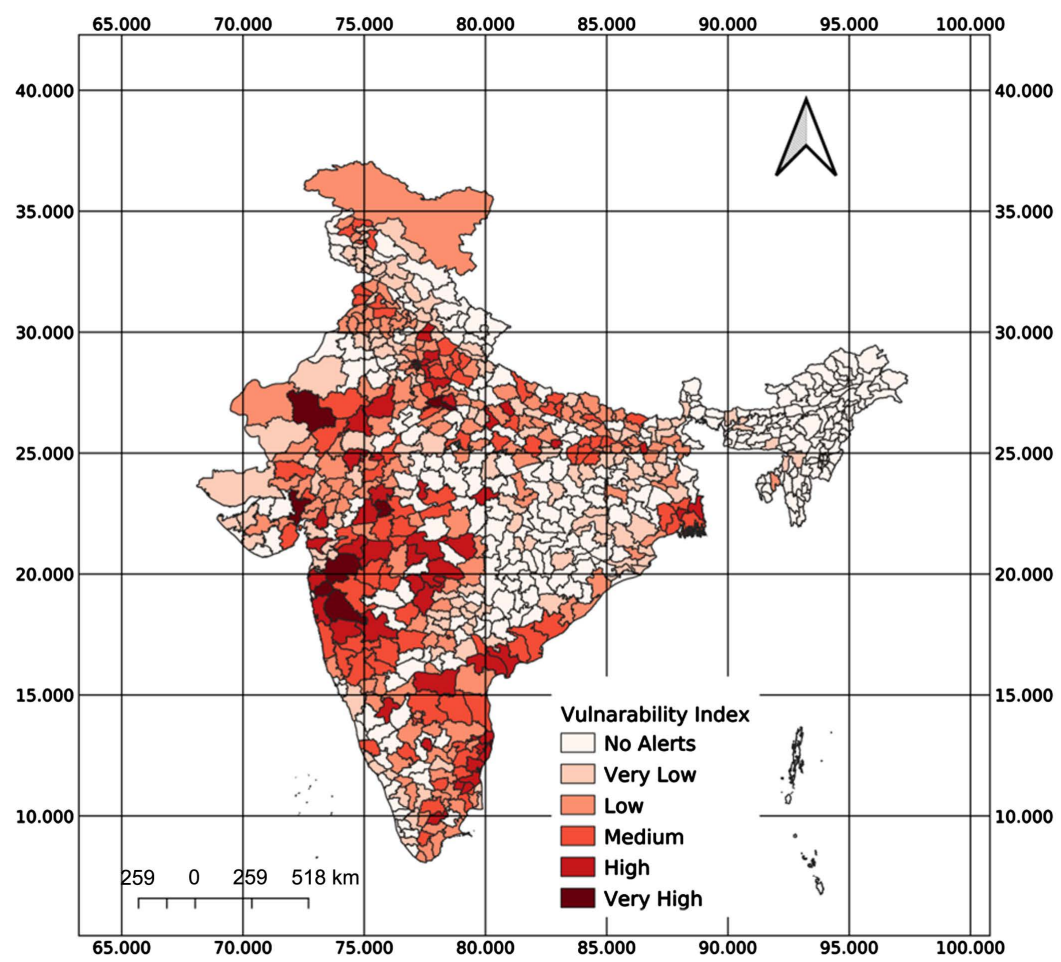

Figure 6. Vulnerability Index of India as on May 2020. 
Distribution across the country for the alerts is depicted in Table 2. It can be clearly observed that nearly $47 \%$ of the districts do not have any alerts issued against them, nearly $13 \%$ of total districts depict either medium high alerts issued against them. The remaining is shared between low alerts and high alerts. The districts with very high alerts issued against them are mainly cities which are highly populated or have had large number of issues in following social distancing.

Total 15 Districts have very high vulnerability risk. These are mainly highly populated areas where the breach in social distancing has been reported via crowdsourced platform. Some of the major locations are within the central part of Mumbai, New Delhi, Ahmedabad. These districts are also declared as Red Zones for containment of COVID-19. Table 3 depicts the total number of lockdown issues pulled from the MapMyIndia's corona Geoportal. It can be clearly observed that the states like Delhi and Maharashtra have the higher number of social distancing breaching issues being reported, thus the districts of the corresponding states have high alerts issued against them.

\subsection{Web GIS Dashboard for Alert Dissemination}

Currently, three web applications have been specifically designed and integrated on the top of the main COVID-19 dashboard to support decision making activity using geospatial tools and services. The dashboard designed for the state of

Table 2. Distribution of count alerts in various districts in India.

\begin{tabular}{cc}
\hline Type of Alerts & Number of Districts \\
\hline No Alerts & 311 \\
Very Low & 154 \\
Low & 53 \\
Medium & 102 \\
High & 99 \\
Very High & 15 \\
\hline
\end{tabular}

Table 3. Reported lockdown issues for different states as on 07 May 2020.

\begin{tabular}{cccc}
\hline State & Lockdown Issues & State & Lockdown Issues \\
\hline Jammu \& Kashmir & 1 & Madhya Pradesh & 5 \\
Himachal Pradesh & 2 & Andhra Pradesh & 3 \\
Chandigarh & 1 & Tamil Nadu & 4 \\
Delhi & 39 & Karnataka & 5 \\
Rajasthan & 3 & Goa & 2 \\
Uttar Pradesh & 8 & Telangana & 1 \\
Bihar & 4 & Maharashtra & 9 \\
West Bengal & 4 & Gujarat & 1
\end{tabular}


Assam can provide the details of schools in spatial domain which may be suitable for quarantine camp in worst case scenario. On the other hand, the Meghalaya dashboard application receives live data from the tracking mobile app developed by Indian Institute of Technology Bombay and provides the visualization in Geo-Xplorer with GIS functionalities. The large scale geospatial database prepared under for Shillong municipality is being used for household identification of the suspected corona affected people. The dashboard of Tripura provides the live visualization of geotagged locations of suspected patients captured during the survey by the Health workers. The layout of the GeoXplorer of Shillong dashboard application is depicted in Figure 7. The dashboard is utilized to visualize the forecast, hot-spots and the current situation of COVID-19 across the country. It is equipped with dynamic charting libraries and visualization tools, to provide quick and holistic information about current and future COVID-19 scenario.

\section{Conclusion}

It is very challenging to model and predict the COVID-19 outbreak accurately, being an infection disease which has many unknown parameters governing to its outbreak. Country's policy and social responsibility largely influence the outbreak spreads. The current study is a two-phase approach to assess outbreak on COVID-19 in India. Phase I addresses the impact of lockdown in outbreak of

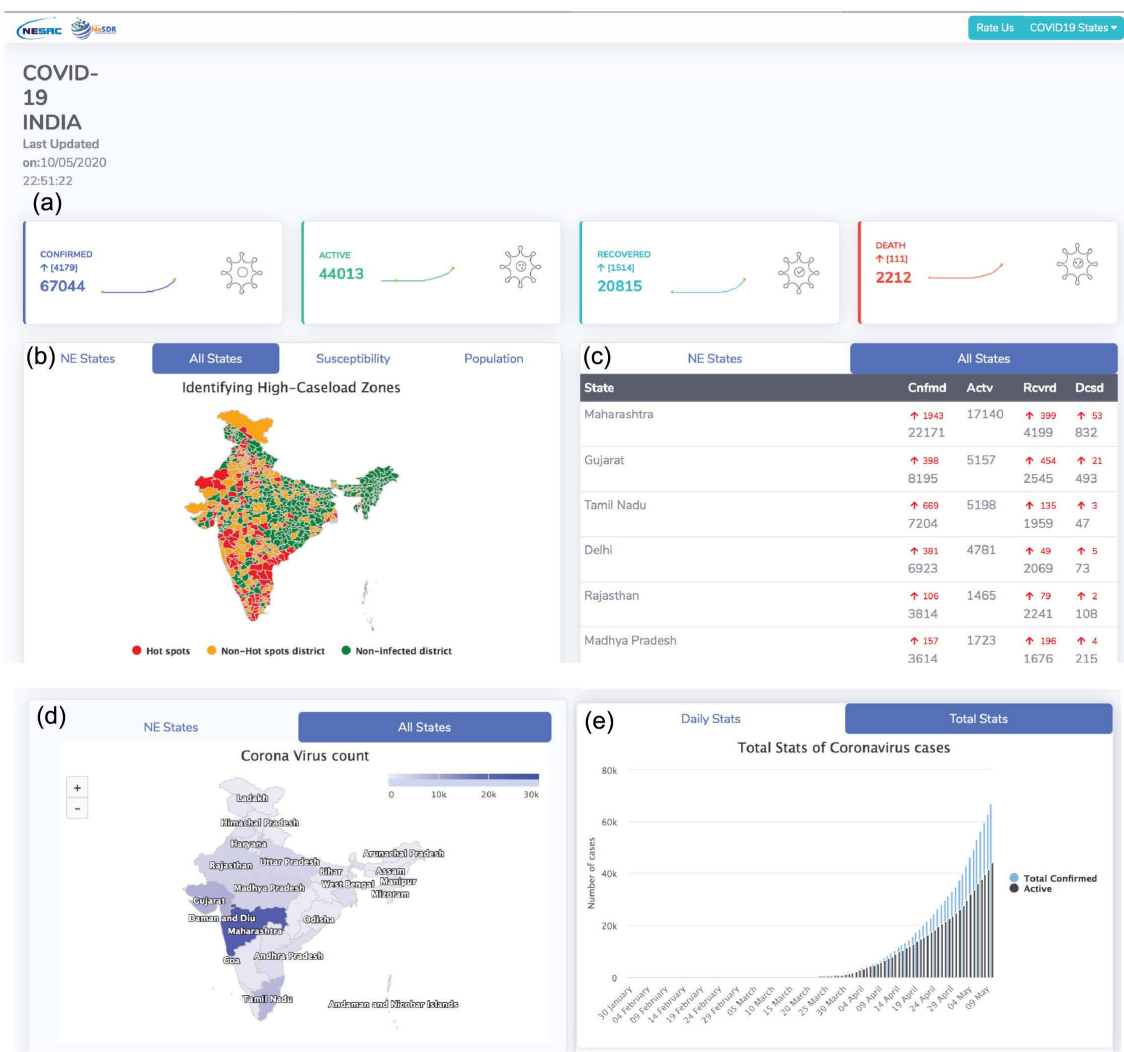

Figure 7. The web application dashboard for COVID-19 India data. 
COVID-19 in states of India with major outbreaks and phase II addresses the objective of issuing alerts for various districts across the country based on the calculated vulnerability index. The model is implemented for understanding the projections under the lockdown scenario i.e. until 31 May 2020. The dynamic parameters of SIR model have increased due to the implementation of unlock (1 June 2020). As a result it is not possible to quantify the breach in the lockdown and movement of passengers. The model forecast for the three scenarios can be further improved by initializing the model parameters using artificial intelligence. Staggered relaxation in lockdown is thus proposed to ensure the containment of the pandemic. We observed that many states are transitioning from strict lockdown to moderate lockdown. Controlled lockdown implementation can reinstate the state with strict lockdown scenario and highly curb the outbreak of the disease. The finding reported here is quite encouraging; however, this may be used as reference for any kind of operational activity as this is exclusively based on the crowdsourced data.

\section{Acknowledgements}

Authors would like to acknowledge the volunteers from Covid-19 India platform who have published the crowd sourced database for public use.

\section{Conflicts of Interest}

The authors declare no conflicts of interest regarding the publication of this paper.

\section{References}

[1] World Health Organization (2020) Coronavirus Disease 2019 (COVID-19) Situation Report-47.

https://www.who.int/docs/default-source/coronaviruse/situation-reports/20200307sitrep-47-covid-19.pdf?sfvrsn=27c364a 4 2

[2] Kumar, P., Singh, R.K., Nanda, C., Kalita, H., Patairiya, S., Sharma, Y.D., Rani, M. and Bhagavathula, A.S. (2020) Forecasting COVID-19 Impact in India Using Pandemic Waves Nonlinear Growth Models. https://doi.org/10.1101/2020.03.30.20047803

[3] Corman, V.M., Landt, O., Kaiser, M., et al. (2020) Detection of 2019 Novel Coronavirus (2019 nCoV) by Real-Time RT-PCR. Euro Surveillance, 25, 23-30. https://doi.org/10.2807/1560-7917.ES.2020.25.3.2000045

[4] Ma, J.L., Dushoff, J., Bolker, B.M. and Earn, D.J.D. (2014) Estimating Initial Epidemic Growth Rates. Bulletin of Mathematical Biology, 76, 245-260. https://doi.org/10.1007/s11538-013-9918-2

[5] Trajectory of World COVID-19 Confirmed Cases. https://aatishb.com/covidtrends/

[6] Hethcote, H.W. (2000) The Mathematics of Infectious Diseases. SIAM Review, 42, 599-653. https://doi.org/10.1137/S0036144500371907

[7] Wu, Z.Y. and McGoogan, J.M. (2020) Characteristics of and Important Lessons from the Coronavirus Disease 2019 (COVID-19) Outbreak in China: Summary of a Report of 72314 Cases from the Chinese Center for Disease Control and Preven- 
tion. JAMA, 23, 1239-1242.

[8] Salman, S. and Salem, M.L. (2020) The Mystery behind Childhood Sparing by COVID-19. International Journal of Cancer and Biomedical Research, 5, 11-13. https://doi.org/10.21608/jcbr.2020.79888

[9] Ranjan, R. (2020) Prediction for COVID-19 Outbreak in India for Epidimological Models. https://doi.org/10.1101/2020.04.02.20051466

[10] Singhal, T.A. (2020) Review of Coronavirus Disease-2019 (COVID-19). The Indian Journal of Pediatrics, 87, 281-286. https://doi.org/10.1007/s12098-020-03263-6

[11] Read, J.M., Bridgen, J.R.E., Cummings, D.A.T., Ho, A. and Jewell, C.P. (2020) Novel Coronavirus 2019-nCoV: Early Estimation of Epidemiological Parameters and Epidemic Predictions. https://doi.org/10.1101/2020.01.23.20018549

[12] Rothe, C., Schunk, M. and Sothmann, P. (2020) Transmission of 2019-nCoV Infection from an Asymptomatic Contact in Germany. New England Journal of Medicine, 382, 970-971. https://doi.org/10.1056/NEJMc2001468

[13] Wu, J.T., Leung, K. and Leung, G.M. (2020) Nowcasting and Forecasting the Potential Domestic and International Spread of the 2019-nCoV Outbreak Originating in Wuhan, China: A Modelling Study. Lancet, 395, 689-697. https://doi.org/10.1016/S0140-6736(20)30260-9

[14] Anastassopoulou, C., Russo, L., Tsakris, A. and Siettos, C. (2020) Data-Based Analysis, Modelling and Forecasting of the COVID-19 Outbreak.

https://doi.org/10.1101/2020.02.11.20022186

\section{Convention Notation}

COVID-19: Corona virus Disease of 2019

SIR: Susceptible Infected and Recovered

SEIR: Susceptible Exposed Infected and Recovered

ODE: Ordinary Differential Equation

RMSE: Root mean square error 